\title{
MODELLING OF DYNAMICS OF A WHEELED MOBILE ROBOT WITH MECANUM WHEELS WITH THE USE OF LAGRANGE EQUATIONS OF THE SECOND KIND
}

\author{
Z. HENDZEL and Ł. RYKAŁA* \\ Department of Applied Mechanics and Robotics \\ Faculty of Mechanical Engineering and Aeronautics \\ Rzeszow University of Technology \\ al. Powstańców Warszawy 12, 35-959 Rzeszów, POLAND \\ E-mails: zenhen@prz.edu.pl; lrykala@prz.edu.pl
}

\begin{abstract}
The work presents the dynamic equations of motion of a wheeled mobile robot with mecanum wheels derived with the use of Lagrange equations of the second kind. Mecanum wheels are a new type of wheels used in wheeled mobile robots and they consist of freely rotating rollers attached to the circumference of the wheels. In order to derive dynamic equations of motion of a wheeled mobile robot, the kinetic energy of the system is determined, as well as the generalised forces affecting the system. The resulting mathematical model of a wheeled mobile robot was generated with the use of Maple V software. The results of a solution of inverse and forward problems of dynamics of the discussed object are also published.
\end{abstract}

Key words: wheeled mobile robots, mecanum wheels, mathematical model.

\section{Introduction}

The new type of wheels used in wheeled mobile robots (abbreviated as WMR) includes the so-called omnidirectional wheels, which consist of a hub and a specific number of rollers installed on the circumference of the hub (Fig.1) $[1,2,3,4,5]$. The mentioned number of rollers can vary between different designs of the wheels, but each of the rollers can freely rotate around its own axis. The wheels can be characterised by means of angle $\delta$ occuring between the axis of own rotation of the wheel and the axis of rotation of the roller (Fig.1), which is equal in mecanum wheels $\delta=45^{\circ}$ [6].

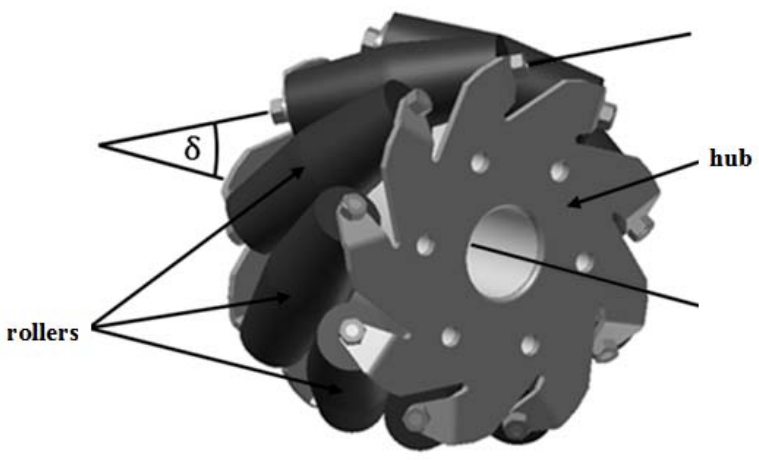

Fig.1. Model of a mecanum wheel with graphically marked angle $\delta$.

\footnotetext{
* To whom correspondence should be addressed
} 
The WMR considered consists of a platform, four identical mecanum wheels and four independently controlled direct-current motors. The mecanum wheels are in turn rigidly placed on the shafts of the particular motors. The virtual model of the mentioned WMR is presented in Fig.2.

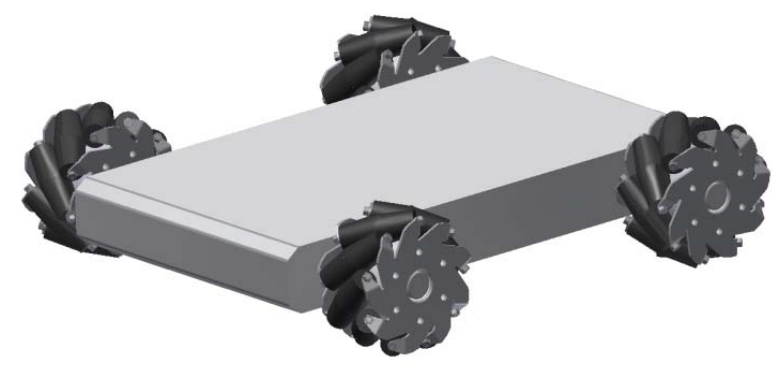

Fig.2. Virtual model of a WMR with mecanum wheels.

It has been assumed in the current configuration that the mecanum wheels located on the opposite part of the WMR have identically oriented angles $\delta$ (Fig.2), which is also presented schematically in Fig.3.

\section{Description of kinematics of a WMR with mecanum wheels}

During movement of the WMR, the mecanum wheels rotate with angular velocity $\dot{\varphi}_{l}$, where $i$ denotes the number of the wheel $(i=1,2,3,4)$. The radiuses of mecanum wheels $R$ and rollers $r$ are constant values that are equal for each of the four wheels. Further assumptions in regard to the WMR are presented graphically in Fig.3. It has also been assumed that the angle of rotation of the platform of the WMR around the characteristic point $S$ is angle $\beta$. The width of the platform is $2 s_{y}$, and the distances between point $S$, and the midpoints of the front and back axle (points $A_{1}, A_{2}$ ) are in both cases equal to $s_{x}$. Furthermore, we assume that all the mecanum wheels are moving on a level base without skidding.

A WMR object with mecanum wheels can be described in a co-ordinate system $x_{p} y_{p} z_{p}$ (Fig.3) connected with the mass centre of the WMR platform, i.e., the characteristic point $S$.

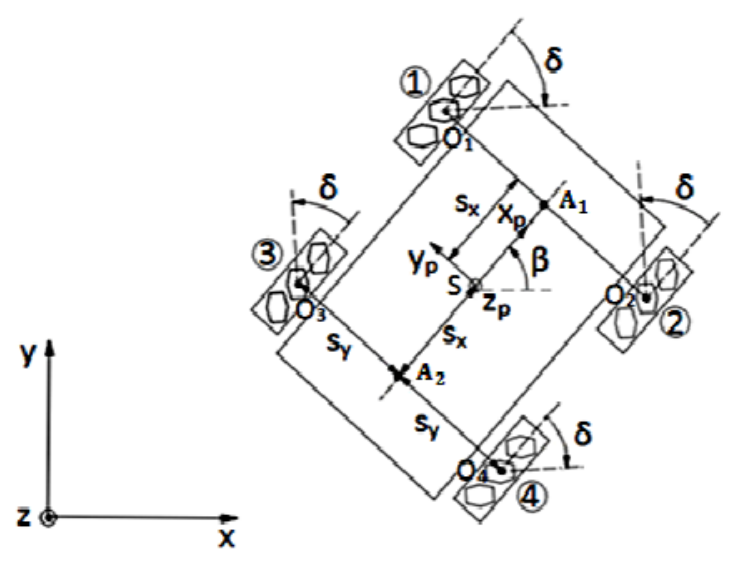

Fig.3. Analytical model of a WMR with mecanum wheels.

The description of kinematics of the WMR in the case under consideration can also be presented in the form of the following equations $[6,7]$

$$
v_{S x_{p}}-v_{S y_{p}}-\dot{\beta}\left(s_{x}+s_{y}\right)-\dot{\varphi}_{l}(R+r)=0 \text {, }
$$




$$
\begin{aligned}
& v_{S x_{p}}+v_{S y_{p}}+\dot{\beta}\left(s_{x}+s_{y}\right)-\dot{\varphi}_{2}(R+r)=0, \\
& v_{S x_{p}}+v_{S y_{p}}-\dot{\beta}\left(s_{x}+s_{y}\right)-\dot{\varphi}_{3}(R+r)=0, \\
& v_{S x_{p}}-v_{S y_{p}}+\dot{\beta}\left(s_{x}+s_{y}\right)-\dot{\varphi}_{4}(R+r)=0
\end{aligned}
$$

where: $v_{S x_{p}}$ - projection of velocity of point $S$ on the $x_{p}$ axis, $v_{S y_{p}}$ - projection of velocity of point $S$ on the $y_{p}$ axis.

Equations (2.1), (2.2), (2.3), (2.4) are equations of holonomic (geometric) constraints. Therefore, the WMR described with the use of the mentioned kinematic equations, is a holonomic object.

For this case it is also possible to present a solution of an inverse kinematics problem assuming the track of point $\mathrm{S}$ and its velocity $\bar{v}_{S}$ in the form of the following relationships

$$
\begin{aligned}
& \dot{\varphi}_{1}=\frac{1}{(R+r)}\left[v_{S x_{p}}-v_{S y_{p}}-\dot{\beta}\left(s_{x}+s_{y}\right)\right], \\
& \dot{\varphi}_{2}=\frac{1}{(R+r)}\left[v_{S x_{p}}+v_{S y_{p}}+\dot{\beta}\left(s_{x}+s_{y}\right)\right], \\
& \dot{\varphi}_{3}=\frac{1}{(R+r)}\left[v_{S x_{p}}+v_{S y_{p}}-\dot{\beta}\left(s_{x}+s_{y}\right)\right], \\
& \dot{\varphi}_{4}=\frac{1}{(R+r)}\left[v_{S x_{p}}-v_{S y_{p}}+\dot{\beta}\left(s_{x}+s_{y}\right)\right] .
\end{aligned}
$$

Equations (2.5), (2.6), (2.7), (2.8) can be presented in the form of a relationship presented below

$$
\dot{\varphi}=J V_{s p}
$$

where

$$
\begin{aligned}
& \dot{\varphi}=\left[\begin{array}{c}
\dot{\varphi}_{1} \\
\dot{\varphi}_{2} \\
\dot{\varphi}_{3} \\
\dot{\varphi}_{4}
\end{array}\right], \\
& J=\left[\begin{array}{ccc}
\frac{1}{(R+r)} & \frac{-1}{(R+r)} & \frac{-\left(s_{x}+s_{y}\right)}{(R+r)} \\
\frac{1}{(R+r)} & \frac{1}{(R+r)} & \frac{\left(s_{x}+s_{y}\right)}{(R+r)} \\
\frac{1}{(R+r)} & \frac{1}{(R+r)} & \frac{-\left(s_{x}+s_{y}\right)}{(R+r)} \\
\frac{1}{(R+r)} & \frac{-1}{(R+r)} & \frac{\left(s_{x}+s_{y}\right)}{(R+r)}
\end{array}\right],
\end{aligned}
$$




$$
V_{s p}=\left[\begin{array}{c}
v_{S x_{p}} \\
v_{S y_{p}} \\
\dot{\beta}
\end{array}\right] .
$$

In order to solve a forward kinematics problem, an inversion operation of matrix $J$ should be performed. Matrix $J$ is a rectangular matrix, therefore in order to perform its inversion one can use the Moore-Penrose theorem on inversion of rectangular matrices. Once the mentioned theorem is applied, the following relationship is obtained [8]

$$
J_{o d}=J^{+} \dot{\varphi} J^{+}=\left(J^{T} J\right)^{-1} J^{T}
$$

where matrix $J^{+}$is a pseudoinverse matrix (Moore-Penrose pseudoinverse) in relation to matrix $J$.

Matrix $J_{o d}$ calculated in accordance with relationship (2.13) is presented in the following matrix form

$$
J_{o d}=\frac{1}{4}\left[\begin{array}{rlll}
(R+r) & (R+r) & (R+r) & (R+r) \\
-(R+r) & (R+r) & (R+r) & -(R+r) \\
\frac{-(R+r)}{\left(s_{x}+s_{y}\right)} & \frac{(R+r)}{\left(s_{x}+s_{y}\right)} & \frac{-(R+r)}{\left(s_{x}+s_{y}\right)} & \frac{(R+r)}{\left(s_{x}+s_{y}\right)}
\end{array}\right] .
$$

The solution of a forward kinematics problem can be received from the following relationship

$$
V_{s p}=J_{o d} \dot{\varphi}
$$

Relationship (2.15) is a source for the following equations

$$
\begin{aligned}
& v_{S x_{p}}=\left(\frac{R+r}{4}\right)\left[\dot{\varphi}_{1}+\dot{\varphi}_{2}+\dot{\varphi}_{3}+\dot{\varphi}_{4}\right], \\
& v_{S y_{p}}=\left(\frac{R+r}{4}\right)\left[-\dot{\varphi}_{1}+\dot{\varphi}_{2}+\dot{\varphi}_{3}-\dot{\varphi}_{4}\right], \\
& \dot{\beta}=\left(\frac{R+r}{4\left(s_{x}+s_{y}\right)}\right)\left[-\dot{\varphi}_{1}+\dot{\varphi}_{2}-\dot{\varphi}_{3}+\dot{\varphi}_{4}\right] .
\end{aligned}
$$

As proved in [6], a roller is a passive element and assuming the lack of skidding between the roller and the ground it does not affect the motion of a mecanum wheel.

\section{Description of dynamics of a WMR with mecanum wheels}

Lagrangian formalism was used in order to obtain dynamic equations of motion of the WMR. Lagrange equations of the second kind for the described holonomic object can be noted in the following way $[7,9]$ 


$$
\frac{d}{d t}\left(\frac{\partial T}{\partial \dot{q}_{j}}\right)-\frac{\partial T}{\partial q_{j}}=Q_{j}
$$

where: $j=1,2, \ldots, s, T$ - kinetic potential, $q_{j}$ - value of the $j$-th generalised coordinate, $Q_{j}$ - value of the $j$-th generalised force, $s$ - number of degrees of the system's freedom.

Afterwards, it was assumed that the WMR moves on a level surface, therefore the kinetic potential $T$ appearing in Lagrange Eq.(3.1) can be presented in the following way

$$
T=E_{k}
$$

where: $E_{k}$ - kinetic energy of the system.

Due to the above-mentioned fact that rollers in the system under analysis are passive elements, the influence of the rollers on the dynamics of the analysed object is omitted in further deliberations, i.e., the kinetic energy of the rollers, the mass of the rollers, and the mass moment of inertia related to the mentioned element are omitted.

Considering the foregoing, the motion of the WMR is related to the motion of its components, i.e., the WMR platform and mecanum wheels. The WMR platform moves in plane motion, whereas the mecanum wheels move in complex motion [7, 10, 11, 12, 13].

The kinetic energy of the WMR platform $E_{k p}$ can be expressed in the following way

$$
E_{k p}=\frac{1}{2} m_{p}\left(v_{S x_{p}}^{2}+v_{S y_{p}}^{2}\right)+\frac{1}{2} I_{p_{z p}} \dot{\beta}^{2}
$$

where: $m_{p}$ - mass of the platform, $I_{p_{z p}}$ - mass moment of inertia of the WMR platform determined in relation to the $z_{p}$ axis, of the $x_{p} y_{p} z_{p}$ co-ordinate system, passing through point $S$.

The mecanum wheels in turn move in complex motion in consequence of the rotary motion of wheels determined around the axis of own rotation of the mecanum wheels and the plane lifting motion of the WMR platform. Therefore, the kinetic energy of the wheels $E_{k m}$ can be expressed in the following way

$$
E_{k m}=\frac{1}{2} I_{k}\left(\dot{\varphi}_{1}^{2}+\dot{\varphi}_{2}^{2}+\dot{\varphi}_{3}^{2}+\dot{\varphi}_{4}^{2}\right)+4\left[\frac{1}{2} m_{k}\left(v_{S x_{p}}^{2}+v_{S y_{p}}^{2}\right)+\frac{1}{2} I_{k_{z p}} \dot{\beta}^{2}\right]
$$

where: $m_{k}$ - mass of a mecanum wheel, $I_{k}$ - mass moment of inertia determined in relation to the axis of own rotation of the wheel, $I_{k_{z p}}$ - mass moment of inertia of a mecanum wheel determined in relation of the $z_{p}$ axis, of the $x_{p} y_{p} z_{p}$ coordinate system, with the middle of the axis in point $S$.

The total kinetic energy of the system $E_{k}$ is presented in the form of the following relationship

$$
E_{k}=E_{k p}+E_{k m}
$$

Once the summation operation is performed, relationship (3.5) takes the following form

$$
E_{k}=\frac{1}{2} m_{p c}\left(v_{S x_{p}}^{2}+v_{S y_{p}}^{2}\right)+\frac{1}{2} I_{p c} \dot{\beta}^{2}+\frac{1}{2} I_{k}\left(\dot{\varphi}_{1}^{2}+\dot{\varphi}_{2}^{2}+\dot{\varphi}_{3}^{2}+\dot{\varphi}_{4}^{2}\right)
$$

where: $m_{p c}$ and $I_{p c}$ are in the following relationships 


$$
\begin{aligned}
& m_{p c}=m_{p}+4 m_{k}, \\
& I_{p c}=I_{p_{z p}}+4 I_{k_{z p}} .
\end{aligned}
$$

Next, in order to simplify the notation of the kinetic energy of the system $E_{k}$, ancillary variables are adopted in the form of formulas presented below

$$
\begin{aligned}
& A=\frac{m_{p c}(R+r)^{2}}{8}, \\
& B=\frac{I_{p c}(R+r)^{2}}{16\left(s_{x}+s_{y}\right)^{2}}, \\
& C=I_{k} .
\end{aligned}
$$

After the variables described by means of relationships (3.9), (3.10), (3.11) are introduced in the formula of the kinetic energy of the system (3.6), the relationship describing kinetic energy of the WMR is presented in the form of the following relationship

$$
\begin{aligned}
& E_{k}=\frac{1}{4} A 2 \dot{\varphi}_{1}^{2}+2 \dot{\varphi}_{2}^{2}+2 \dot{\varphi}_{3}^{2}+2 \dot{\varphi}_{4}^{2}+4 \dot{\varphi}_{1} \dot{\varphi}_{4}+4 \dot{\varphi}_{2} \dot{\varphi}_{3}+\frac{1}{2} B \dot{\varphi}_{1}^{2}+\dot{\varphi}_{2}^{2}+ \\
& +\dot{\varphi}_{3}^{2}+\dot{\varphi}_{4}^{2}-2 \dot{\varphi}_{1} \dot{\varphi}_{2}-2 \dot{\varphi}_{3} \dot{\varphi}_{4}+2 \dot{\varphi}_{1} \dot{\varphi}_{3}-2 \dot{\varphi}_{1} \dot{\varphi}_{4}-2 \dot{\varphi}_{2} \dot{\varphi}_{3}+2 \dot{\varphi}_{2} \dot{\varphi}_{4}+ \\
& +\frac{1}{2} C\left[\dot{\varphi}_{1}^{2}+\dot{\varphi}_{2}^{2}+\dot{\varphi}_{3}^{2}+\dot{\varphi}_{4}^{2}\right] .
\end{aligned}
$$

After the notation is simplified and shared factors are factored out, formula (3.12) can be noted as Eq.(3.13)

$$
\begin{aligned}
& E_{k}=\frac{1}{2}(A+B+C)\left[\dot{\varphi}_{1}^{2}+\dot{\varphi}_{2}^{2}+\dot{\varphi}_{3}^{2}+\dot{\varphi}_{4}^{2}\right]+(A-B) \dot{\varphi}_{1} \dot{\varphi}_{4}+ \\
& +\dot{\varphi}_{2} \dot{\varphi}_{3}-B\left[\dot{\varphi}_{1} \dot{\varphi}_{2}+\dot{\varphi}_{3} \dot{\varphi}_{4}-\dot{\varphi}_{1} \dot{\varphi}_{3}-\dot{\varphi}_{2} \dot{\varphi}_{4}\right] .
\end{aligned}
$$

The analysed WMR model has four degrees of freedom, therefore the generalised coordinates are assumed to be the following variables noted in the following form

$$
\left[\begin{array}{l}
q_{1} \\
q_{2} \\
q_{3} \\
q_{4}
\end{array}\right]=\left[\begin{array}{l}
\varphi_{1} \\
\varphi_{2} \\
\varphi_{3} \\
\varphi_{4}
\end{array}\right] .
$$

Figure 4 and Fig. 5 present in turn the forces and moments of forces acting on pairs of mecanum wheels, in succession 2, 4 and 1, 3, taking into account the forces of gravity acting on the WMR platform. In the analysis conducted it has been assumed that the interactions of the pair of elements: engine, mecanum 
wheel are down to the fact that the $\mathrm{i}$-th mecanum wheel is propelled by means of driving moment $\tau_{i}(i=1$, $2,3,4)$ originating from the i-th power transmission system. Furthermore, it has been assumed that the weight of the WMR platform is $\bar{G}_{p}$, whereas the weight of the i-th mecanum wheel is $\bar{G}_{i}$ and it moves on a flat, rough (coefficient of dry friction $\mu_{i}$ ) and deformable (coefficient of rolling friction $f_{i}$ ) surface without skidding. The analysis also takes into account the dry friction force $\bar{T}_{i}$ and the pressure force $\bar{N}_{i}$ for the i-th wheel.

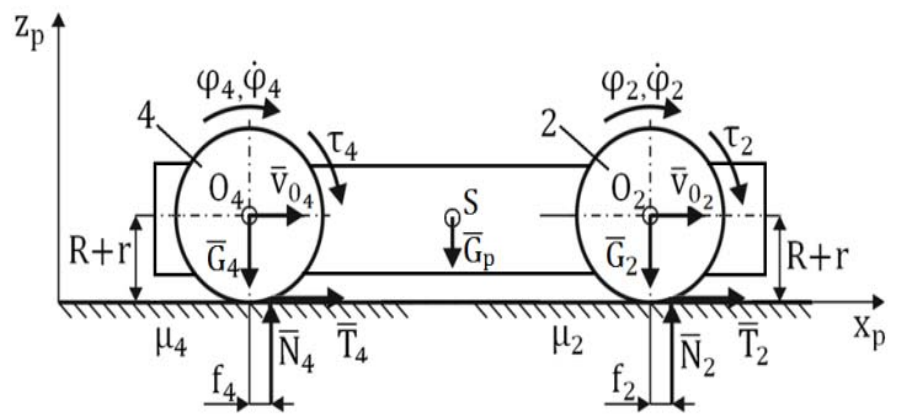

Fig.4. Forces and moments of forces acting on mecanum wheels no. 2 and 4.

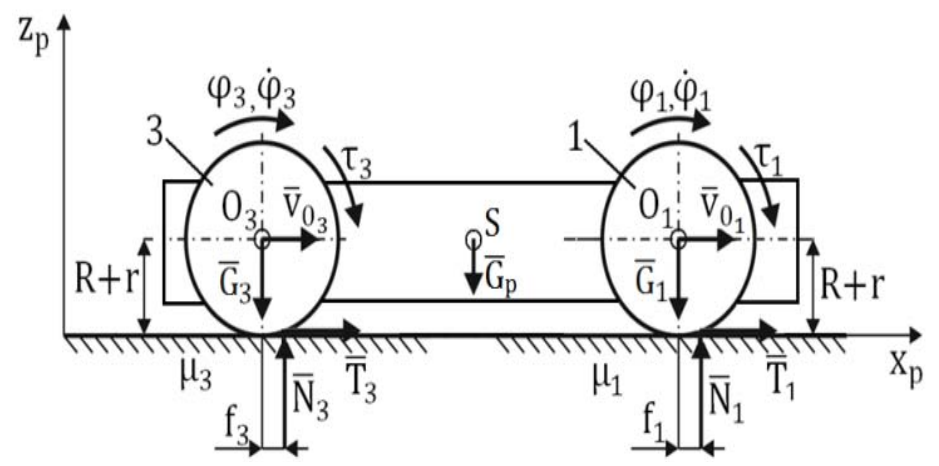

Fig.5. Forces and moments of forces acting on mecanum wheels no. 1 and 3.

Elementary work of the system of forces amounts to

$$
\partial L=\left(\tau_{1}-N_{1} f_{1}\right) \partial \varphi_{1}+\left(\tau_{2}-N_{2} f_{2}\right) \partial \varphi_{2}+\left(\tau_{3}-N_{3} f_{3}\right) \partial \varphi_{3}+\left(\tau_{4}-N_{4} f_{4}\right) \partial \varphi_{4} .
$$

Relationship (3.15) indicates that particular generalised forces satisfy the following equations

$$
\begin{aligned}
& Q_{1}=\tau_{1}-N_{1} f_{1}, \\
& Q_{2}=\tau_{2}-N_{2} f_{2}, \\
& Q_{3}=\tau_{3}-N_{3} f_{3}, \\
& Q_{4}=\tau_{4}-N_{4} f_{4} .
\end{aligned}
$$


The moments of rolling friction appearing in relationships (3.16), (3.17), (3.18), (3.19) are then made dependant on the sense of the value of angular velocity of the wheels $\dot{\varphi}_{i}$ with the use of the signum function

$$
\begin{aligned}
& Q_{1}=\tau_{1}-N_{1} f_{1} \operatorname{sgn}\left(\dot{\varphi}_{1}\right), \\
& Q_{2}=\tau_{2}-N_{2} f_{2} \operatorname{sgn}\left(\dot{\varphi}_{2}\right), \\
& Q_{3}=\tau_{3}-N_{3} f_{3} \operatorname{sgn}\left(\dot{\varphi}_{3}\right), \\
& Q_{4}=\tau_{4}-N_{4} f_{4} \operatorname{sgn}\left(\dot{\varphi}_{4}\right) .
\end{aligned}
$$

Furthermore, the following relationship is correct in the current case, which significantly simplifies formula (3.1)

$$
\frac{\partial T}{\partial \varphi}=0
$$

Considering the foregoing Lagrange Eq.(3.1) can be presented in the form of a matrix

$$
\frac{d}{d t}\left[\begin{array}{ccc}
\frac{1}{2}(A+B+C) \dot{\varphi}_{1}^{2} & (A-B) \dot{\varphi}_{4} & -B\left(\dot{\varphi}_{2}-\dot{\varphi}_{3}\right) \\
\frac{1}{2}(A+B+C) \dot{\varphi}_{2}^{2} & (A-B) \dot{\varphi}_{3} & -B\left(\dot{\varphi}_{1}-\dot{\varphi}_{4}\right) \\
\frac{1}{2}(A+B+C) \dot{\varphi}_{3}^{2} & (A-B) \dot{\varphi}_{2} & -B\left(\dot{\varphi}_{4}-\dot{\varphi}_{1}\right) \\
\frac{1}{2}(A+B+C) \dot{\varphi}_{4}^{2} & (A-B) \dot{\varphi}_{1} & -B\left(\dot{\varphi}_{3}-\dot{\varphi}_{2}\right)
\end{array}\right]=\left[\begin{array}{c}
Q_{1} \\
Q_{2} \\
Q_{3} \\
Q_{4}
\end{array}\right] .
$$

Once a differentiation operation is performed in relation to the matrix time located on the left side of the equal sign in formula (3.25), the relationship can be noted in the following way

$$
M\left[\begin{array}{c}
\ddot{\varphi}_{1} \\
\ddot{\varphi}_{2} \\
\ddot{\varphi}_{3} \\
\ddot{\varphi}_{4}
\end{array}\right]=\left[\begin{array}{c}
Q_{1} \\
Q_{2} \\
Q_{3} \\
Q_{4}
\end{array}\right]
$$

where matrix $\mathrm{M}$ is in the following relationship

$$
M=\left[\begin{array}{llll}
(A+B+C) & (-B) & (B) & (A-B) \\
(-B) & (A+B+C) & (A-B) & (B) \\
(B) & (A-B) & (A+B+C) & (-B) \\
(A-B) & (B) & (-B) & (A+B+C)
\end{array}\right] .
$$


Furthermore, formula (3.26) can be transformed so as to obtain a solution of an inverse dynamics problem, i.e., calculate the relationships describing driving moments $\tau_{i}$ of particular mecanum wheels

$$
\left[\begin{array}{c}
\tau_{1} \\
\tau_{2} \\
\tau_{3} \\
\tau_{4}
\end{array}\right]=M\left[\begin{array}{c}
\ddot{\varphi}_{1} \\
\ddot{\varphi}_{2} \\
\ddot{\varphi}_{3} \\
\ddot{\varphi}_{4}
\end{array}\right]+\left[\begin{array}{c}
N_{1} f_{1} \operatorname{sgn}\left(\dot{\varphi}_{1}\right) \\
N_{2} f_{2} \operatorname{sgn}\left(\dot{\varphi}_{2}\right) \\
N_{3} f_{3} \operatorname{sgn}\left(\dot{\varphi}_{3}\right) \\
N_{4} f_{4} \operatorname{sgn}\left(\dot{\varphi}_{4}\right)
\end{array}\right] .
$$

Relationship (3.26) can also be transformed in order to obtain angular acceleration of the wheels $\ddot{\varphi}_{i}$ $(i=1,2,3,4)$

$$
\left[\begin{array}{c}
\ddot{\varphi}_{1} \\
\ddot{\varphi}_{2} \\
\ddot{\varphi}_{3} \\
\ddot{\varphi}_{4}
\end{array}\right]=M^{-1}\left[\begin{array}{c}
\tau_{1}-N_{1} f_{1} \operatorname{sgn}\left(\dot{\varphi}_{1}\right) \\
\tau_{2}-N_{2} f_{2} \operatorname{sgn}\left(\dot{\varphi}_{2}\right) \\
\tau_{3}-N_{3} f_{3} \operatorname{sgn}\left(\dot{\varphi}_{3}\right) \\
\tau_{4}-N_{4} f_{4} \operatorname{sgn}\left(\dot{\varphi}_{4}\right)
\end{array}\right]
$$

where matrix $M^{-1}$ is in the following relationship

$$
M^{-1}=\left[\begin{array}{ccccc}
\frac{2 A B+A C+3 B C+C^{2}}{\left(8 A B+2 A C+4 B C+C^{2}\right) C} & \frac{B}{(4 B+C) C} & \frac{(-B)}{(4 B+C) C} & \frac{-(2 A B+A C-B C)}{\left(8 A B+2 A C+4 B C+C^{2}\right) C} \\
\frac{B}{(4 B+C) C} & \frac{2 A B+A C+3 B C+C^{2}}{\left(8 A B+2 A C+4 B C+C^{2}\right) C} & \frac{-(2 A B+A C-B C)}{\left(8 A B+2 A C+4 B C+C^{2}\right) C} & \frac{(-B)}{(4 B+C) C} \\
\frac{(-B)}{(4 B+C) C} & \frac{-(2 A B+A C-B C)}{\left(8 A B+2 A C+4 B C+C^{2}\right) C} & \frac{2 A B+A C+3 B C+C^{2}}{\left(8 A B+2 A C+4 B C+C^{2}\right) C} & \frac{B}{(4 B+C) C} \\
\frac{-(2 A B+A C-B C)}{\left(8 A B+2 A C+4 B C+C^{2}\right) C} & \frac{(-B)}{(4 B+C) C} & \frac{B}{(4 B+C) C} & \frac{2 A B+A C+3 B C+C^{2}}{\left(8 A B+2 A C+4 B C+C^{2}\right) C}
\end{array}\right]
$$

The analysed object can also be described in a stationary coordinate system $x y z$ (Fig.3). When describing the object in an $x y z$ coordinate system, one can use the homogeneous transformation matrixes and note the following equations

$$
\begin{aligned}
& v_{S x_{p}}=\dot{x}_{S} \cos \beta+\dot{y}_{S} \sin \beta, \\
& v_{S y_{p}}=-\dot{x}_{S} \sin \beta+\dot{y}_{S} \cos \beta .
\end{aligned}
$$

After differentiation with respect to time of Eqs (3.31), (3.32) and Eqs (2.16), (2.17), (2.18), and then by means of equating them to each other, the following three relationships are obtained

$$
\ddot{x}_{S} \cos \beta-\dot{x}_{S} \dot{\beta} \sin \beta+\ddot{y}_{S} \sin \beta+\dot{y}_{S} \dot{\beta} \cos \beta=\left(\frac{(R+r)}{4}\right)\left[\ddot{\varphi}_{1}+\ddot{\varphi}_{2}+\ddot{\varphi}_{3}+\ddot{\varphi}_{4}\right],
$$




$$
\begin{aligned}
& -\ddot{x}_{S} \sin \beta-\dot{x}_{S} \dot{\beta} \cos \beta+\ddot{y}_{S} \cos \beta-\dot{y}_{S} \dot{\beta} \sin \beta=\left(\frac{(R+r)}{4}\right)\left[-\ddot{\varphi}_{1}+\ddot{\varphi}_{2}+\ddot{\varphi}_{3}-\ddot{\varphi}_{4}\right], \\
& \ddot{\beta}=\left(\frac{(R+r)}{4\left(s_{x}+s_{y}\right)}\right)\left[-\ddot{\varphi}_{1}+\ddot{\varphi}_{2}-\ddot{\varphi}_{3}+\ddot{\varphi}_{4}\right] .
\end{aligned}
$$

By means of introduction of angular accelerations of wheels $\ddot{\varphi}_{i}(i=1,2,3,4)$ resulting from matrix relationship (3.29) in Eqs (3.33), (3.34), (3.35), a dynamic equation of motion of the WMR is obtained in the form of the following relationships

$$
\begin{aligned}
& \ddot{x}_{S} \cos \beta-\dot{x}_{S} \dot{\beta} \sin \beta+\ddot{y}_{S} \sin \beta+\dot{y}_{S} \dot{\beta} \cos \beta=\frac{(R+r)}{4(2 A+C)}\left[\tau_{1}+\tau_{2}+\tau_{3}+\tau_{4}+\right. \\
& \left.-N_{1} f_{1} \operatorname{sgn}\left(\dot{\varphi}_{1}\right)-N_{2} f_{2} \operatorname{sgn}\left(\dot{\varphi}_{2}\right)-N_{3} f_{3} \operatorname{sgn}\left(\dot{\varphi}_{3}\right)-N_{4} f_{4} \operatorname{sgn}\left(\dot{\varphi}_{4}\right)\right], \\
& -\ddot{x}_{S} \sin \beta-\dot{x}_{S} \dot{\beta} \cos \beta+\ddot{y}_{S} \cos \beta-\dot{y}_{S} \dot{\beta} \sin \beta=\frac{-(R+r)}{4(2 A+C)}\left[\tau_{1}-\tau_{2}-\tau_{3}+\tau_{4}+\right. \\
& \left.-N_{1} f_{1} \operatorname{sgn}\left(\dot{\varphi}_{1}\right)+N_{2} f_{2} \operatorname{sgn}\left(\dot{\varphi}_{2}\right)+N_{3} f_{3} \operatorname{sgn}\left(\dot{\varphi}_{3}\right)-N_{4} f_{4} \operatorname{sgn}\left(\dot{\varphi}_{4}\right)\right], \\
& \ddot{\beta}=\frac{-(R+r)}{4\left(s_{x}+s_{y}\right)(4 B+C)} \tau_{1}-\tau_{2}+\tau_{3}-\tau_{4}-N_{1} f_{1} \operatorname{sgn}\left(\dot{\varphi}_{1}\right)+ \\
& +N_{2} f_{2} \operatorname{sgn}\left(\dot{\varphi}_{2}\right)-N_{3} f_{3} \operatorname{sgn}\left(\dot{\varphi}_{3}\right)+N_{4} f_{4} \operatorname{sgn}\left(\dot{\varphi}_{4}\right) .
\end{aligned}
$$

Next, it is assumed that the WMR moves on an identical surface, which means that all the rolling resistance coefficients for particular wheels are equal and in the following relationship

$$
f_{1}=f_{2}=f_{3}=f_{4}=f \text {. }
$$

Once relationship (3.39) is taken into account and the notation of Eqs (3.36), (3.37), (3.38) is ordered, the following relationships are obtained

$$
\begin{aligned}
& \left(m_{p c}+\frac{4 I_{k}}{(R+r)^{2}}\right)\left[\cos \beta\left(\ddot{x}_{S}+\dot{y}_{S} \dot{\beta}\right)+\sin \beta\left(\ddot{y}_{S}-\dot{x}_{S} \dot{\beta}\right)\right]=\left(\frac{1}{(R+r)}\right)\left[\tau_{1}+\tau_{2}+\tau_{3}+\tau_{4}+\right. \\
& \left.-f\left(N_{1} \operatorname{sgn}\left(\dot{\varphi}_{1}\right)+N_{2} \operatorname{sgn}\left(\dot{\varphi}_{2}\right)+N_{3} \operatorname{sgn}\left(\dot{\varphi}_{3}\right)+N_{4} \operatorname{sgn}\left(\dot{\varphi}_{4}\right)\right)\right], \\
& \left(m_{p c}+\frac{4 I_{k}}{(R+r)^{2}}\right)\left[\cos \beta\left(\ddot{y}_{S}-\dot{x}_{S} \dot{\beta}\right)-\sin \beta\left(\ddot{x}_{S}+\dot{y}_{S} \dot{\beta}\right)\right]=\left(\frac{-1}{(R+r)}\right)\left[\tau_{1}-\tau_{2}-\tau_{3}+\tau_{4}+\right. \\
& \left.-f\left(\mathrm{~N}_{l} \operatorname{sgn}\left(\dot{\varphi}_{1}\right)-\mathrm{N}_{2} \operatorname{sgn}\left(\dot{\varphi}_{2}\right)-\mathrm{N}_{3} \operatorname{sgn}\left(\dot{\varphi}_{3}\right)+\mathrm{N}_{4} \operatorname{sgn}\left(\dot{\varphi}_{4}\right)\right)\right],
\end{aligned}
$$




$$
\begin{aligned}
& \left(I_{p c}+4 I_{k} \frac{\left(s_{x}+s_{y}\right)^{2}}{(R+r)^{2}}\right) \ddot{\beta}=\frac{-\left(s_{x}+s_{y}\right)}{(R+r)}\left[\tau_{1}-\tau_{2}+\tau_{3}-\tau_{4}+\right. \\
& \left.-f\left(\mathrm{~N}_{1} \operatorname{sgn}\left(\dot{\varphi}_{1}\right)-\mathrm{N}_{2} \operatorname{sgn}\left(\dot{\varphi}_{2}\right)+\mathrm{N}_{3} \operatorname{sgn}\left(\dot{\varphi}_{3}\right)-\mathrm{N}_{4} \operatorname{sgn}\left(\dot{\varphi}_{4}\right)\right)\right] .
\end{aligned}
$$

Figure 6 in turn presents force relationships, i.e., the pressure forces of particular wheels on the base $\bar{N}_{i}$, the weight of particular wheels of the WMR $\bar{G}_{i}$ and the weight of the platform of the WMR $\bar{G}_{p}$ in a two-dimensional projection to axes $x_{p}, z_{p}$. Furthermore, it is assumed that the centre of gravity of the WMR overlaps the geometric centre of the WMR platform, i.e., point $S$.

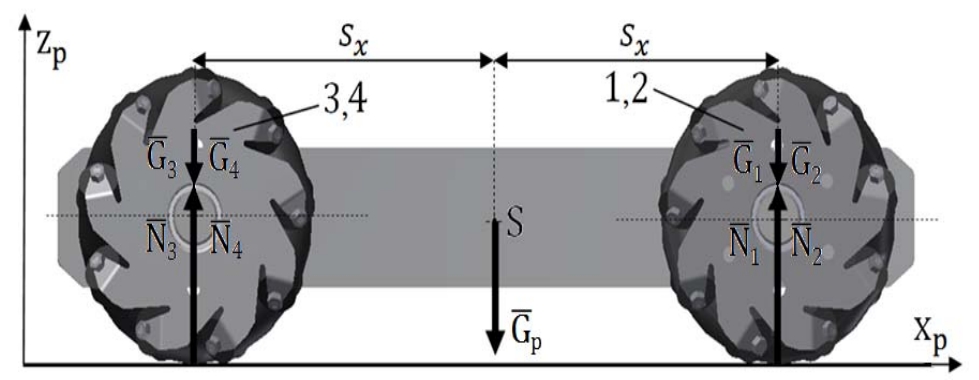

Fig.6. Force relationships of the WMR model.

In order to determine the values of the pressure forces $\bar{N}_{i}$ one needs to analyse the case of equilibrium of the WMR positioned in accordance with Fig.6. In the discussed system, there are no forces acting in the direction of the $x_{p}$ axis. By means of projecting the present forces to the $z_{p}$ axis, it is possible to note the following equation

$$
N_{1}+N_{2}+N_{3}+N_{4}-G_{p}-G_{1}-G_{2}-G_{3}-G_{4}=0 .
$$

The next equation can be obtained by means of calculating the general moment of the force system in relationship to point $S$.

$$
\left(N_{1}+N_{2}\right) s_{x}-\left(N_{3}+N_{4}\right) s_{x}+\left(G_{3}+G_{4}\right) s_{x}-\left(G_{1}+G_{2}\right) s_{x}=0 .
$$

It was then assumed that all the wheels are of the same weight equal to $G_{k}=G_{1}=G_{2}=G_{3}=G_{4}$ and that the total weight of the WMR is equal to $G_{r}=G_{p}+4 G_{k}$. Therefore, Eqs (3.43) and (3.44) can be presented in the following form

$$
\begin{aligned}
& N_{1}+N_{2}+N_{3}+N_{4}-G_{r}=0, \\
& \left(N_{1}+N_{2}\right) s_{x}-\left(N_{3}+N_{4}\right) s_{x}=0 .
\end{aligned}
$$

Assuming in addition that $N_{1}=N_{2}$ and $N_{3}=N_{4}$, it is possible to solve the system of Eqs (3.45), (3.46) and obtain the following relationship

$$
N_{1}=N_{2}=N_{3}=N_{4}=\frac{G_{r}}{4}
$$


By means of introducing relationship (3.47) in Eqs (3.40), (3.41), (3.42) the following equations are obtained

$$
\begin{aligned}
& \left(m_{p c}+\frac{4 I_{k}}{(R+r)^{2}}\right)\left[\cos \beta\left(\ddot{x}_{S}+\dot{y}_{S} \dot{\beta}\right)+\sin \beta\left(\ddot{y}_{S}-\dot{x}_{S} \dot{\beta}\right)\right]= \\
& =\left(\frac{1}{(\mathrm{R}+\mathrm{r})}\right)\left[\tau_{1}+\tau_{2}+\tau_{3}+\tau_{4}-\frac{G_{r} f}{4}\left(\operatorname{sgn}\left(\dot{\varphi}_{1}\right)+\operatorname{sgn}\left(\dot{\varphi}_{2}\right)+\operatorname{sgn}\left(\dot{\varphi}_{3}\right)+\operatorname{sgn}\left(\dot{\varphi}_{4}\right)\right)\right] \\
& \left(m_{p c}+\frac{4 I_{k}}{(R+r)^{2}}\right)\left[\cos \beta\left(\ddot{y}_{S}-\dot{x}_{S} \dot{\beta}\right)-\sin \beta\left(\ddot{x}_{S}+\dot{y}_{S} \dot{\beta}\right)\right]= \\
& =\left(\frac{-1}{(\mathrm{R}+\mathrm{r})}\right)\left[\tau_{1}-\tau_{2}-\tau_{3}+\tau_{4}-\frac{G_{r} f}{4}\left(\operatorname{sgn}\left(\dot{\varphi}_{1}\right)-\operatorname{sgn}\left(\dot{\varphi}_{2}\right)-\operatorname{sgn}\left(\dot{\varphi}_{3}\right)+\operatorname{sgn}\left(\dot{\varphi}_{4}\right)\right)\right] \\
& \left(I_{p c}+4 I_{k} \frac{\left(s_{x}+s_{y}\right)^{2}}{(R+r)^{2}}\right) \ddot{\beta}= \\
& =\frac{-\left(s_{x}+s_{y}\right)}{(R+r)}\left[\tau_{1}-\tau_{2}+\tau_{3}-\tau_{4}-\frac{G_{r} f}{4}\left(\operatorname{sgn}\left(\dot{\varphi}_{1}\right)-\operatorname{sgn}\left(\dot{\varphi}_{2}\right)+\operatorname{sgn}\left(\dot{\varphi}_{3}\right)-\operatorname{sgn}\left(\dot{\varphi}_{4}\right)\right)\right]
\end{aligned}
$$

The defined Eqs (3.48), (3.49), (3.50) describing the dynamics of the object can be used in the analysis of an inverse dynamics problem during the synthesis of control algorithm, and as a forward dynamics problem during simulation studies of motion control algorithms for a wheeled mobile robot with mecanum wheels.

\section{Numerical simulations}

The analysis of the dynamics of the object presented in the previous chapter was used to perform numerical simulations for the solution of an inverse dynamics problem of a WMR with mecanum wheels, and then the forward dynamics problem in order to perform numerical verification of correctness of the obtained dynamic equations of motion. The studies have been performed in the Matlab/Simulink environment. The following geometric dimensions of the WMR have been adopted in the simulations: $R=0.035[\mathrm{~m}], r=0.015[\mathrm{~m}], s_{y}=0.158[\mathrm{~m}], s_{x}=0.095[\mathrm{~m}]$. The simulations also assumed the approximation of the adopted profile of velocity of the characteristic point of the WMR in the form of the following relationship

$$
v_{S}=v_{u s t}\left(\frac{1}{1+e^{-c\left(t-t_{r}\right)}}-\frac{1}{1+e^{-c\left(t-t_{h}\right)}}\right)
$$

where: $v_{u s t}[\mathrm{~m} / \mathrm{s}]$ is the setpoint velocity of the characteristic point of the WMR in stationary state, $c[1 / \mathrm{s}]$ is the coefficient affecting the rate of change of velocity at the time of acceleration and breaking, whereas $t_{r}[s]$ and $t_{h}[s]$ are parameters describing average acceleration and braking times. For the purpose of the approximation of the function $f\left(\dot{\varphi}_{i}\right)=\operatorname{sgn}\left(\dot{\varphi}_{i}\right)$ appearing in dynamic equations of motion by means of a continuous function with subsequent continuous derivatives, a bipolar sigmoid function $g\left(\dot{\varphi}_{i}\right)$ is used, described by means of the following relationship 


$$
g\left(\dot{\varphi}_{i}\right)=\frac{2}{1+e^{-10 \dot{\varphi}_{i}}}-1
$$

Figure 7 presents a graphical comparison of behaviours of functions $f\left(\dot{\varphi}_{i}\right)$ and $g\left(\dot{\varphi}_{i}\right)$.

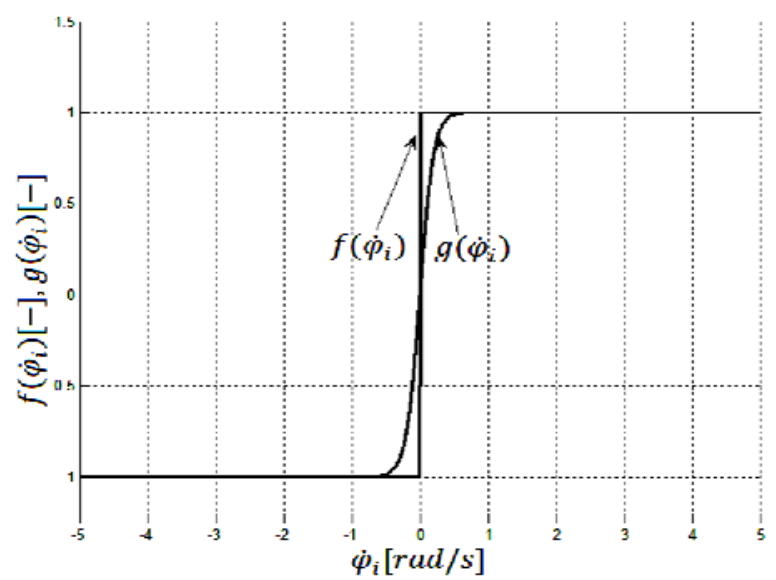

Fig.7. Comparison of behaviours of function $f\left(\dot{\varphi}_{i}\right)$ and $g\left(\dot{\varphi}_{i}\right)$.

In order to describe the mass of the platform and the mass of a single mecanum wheel, the following values have been adopted: $m_{p}=2[\mathrm{~kg}], m_{k}=0.4[\mathrm{~kg}]$. The mass moments of inertia appearing in dynamic equations of motion are equal to: $I_{k}=0.0005\left[\mathrm{~kg} \cdot \mathrm{m}^{2}\right], I_{p_{z p}}=0.0178\left[\mathrm{~kg} \cdot \mathrm{m}^{2}\right], I_{k_{z p}}=0.0139\left[\mathrm{~kg} \cdot \mathrm{m}^{2}\right]$. Next, a coefficient of rolling friction has been adopted, equal to $f=0.002[\mathrm{~m}]$.

\section{Results of numerical simulations}

In the course of numerical studies, a simulation was performed, presenting the motion of the characteristic point of the WMR on a rectilinear trajectory inclined at an angle of $\alpha=\frac{\pi}{6}[\mathrm{rad}]$ in relation to axis $x$ of the $x y z$ coordinate system with non-zero value of angle $\beta(t)=-\frac{\pi}{4}[\mathrm{rad}]$ that was constant in the course of the simulation The position parameters of the initial characteristic point of the WMR in the discussed numerical situation are the following

$$
\begin{array}{ll}
x_{s}(0)=0, & y_{s}(0)=0, \quad \beta(0)=-\frac{\pi}{4}[\mathrm{rad}], \\
\dot{x}_{s}(0)=0, & \dot{y}_{s}(0)=0, \quad \dot{\beta}(0)=0 .
\end{array}
$$

In accordance with the previous description, the trajectory of the motion of the characteristic point of the WMR in this case is described by the following relationship

$$
y_{s}\left(x_{s}\right)=\operatorname{tg}\left(\frac{\pi}{6}\right) x_{s} .
$$


The results of numerical studies for the discussed simulation are presented in Figs 8, 9, 10, 11, 12, $13,14,15,16,17$. The trajectory of motion of the characteristic point of the WMR is presented graphically together with the configuration of the platform of the WMR in Fig.8.

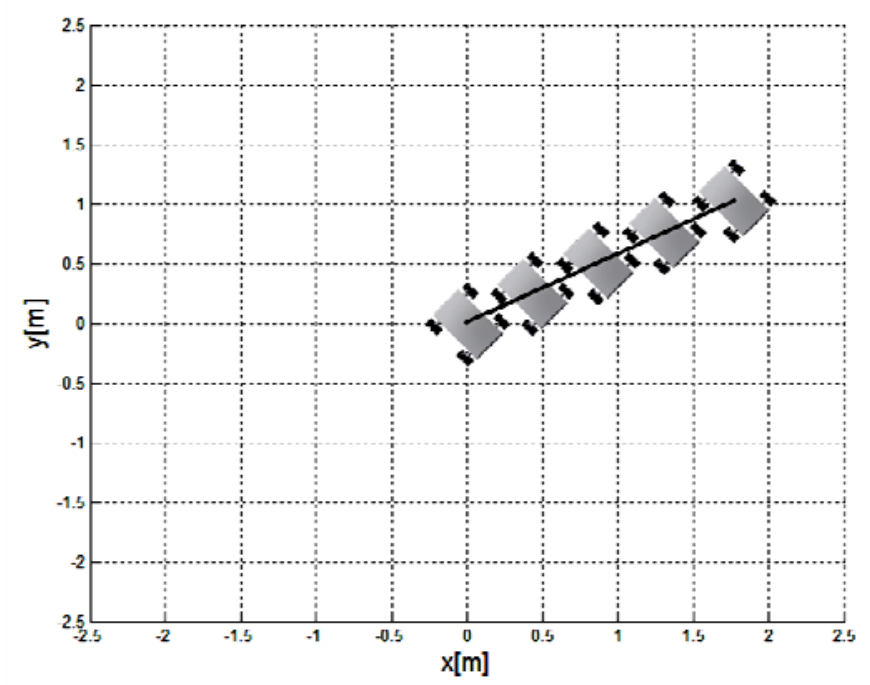

Fig.8. Trajectory of motion of the characteristic point with graphical reflection of configuration of the WMR platform in the course of simulation.

Figure 9 presents the system of coordinates of the characteristic point of the WMR and the angle of rotation of the frame, whereas Fig.10 presents projections of velocity of the characteristic point of the WMR and the angular velocity of the platform of the WMR. Figure 11 in turn illustrates the solution of the inverse kinematics problem for the discussed case of motion of the characteristic point of the WMR, being two behaviours of angular velocity identical to the pairs of wheels no. 1, 4 and 2, 3 .

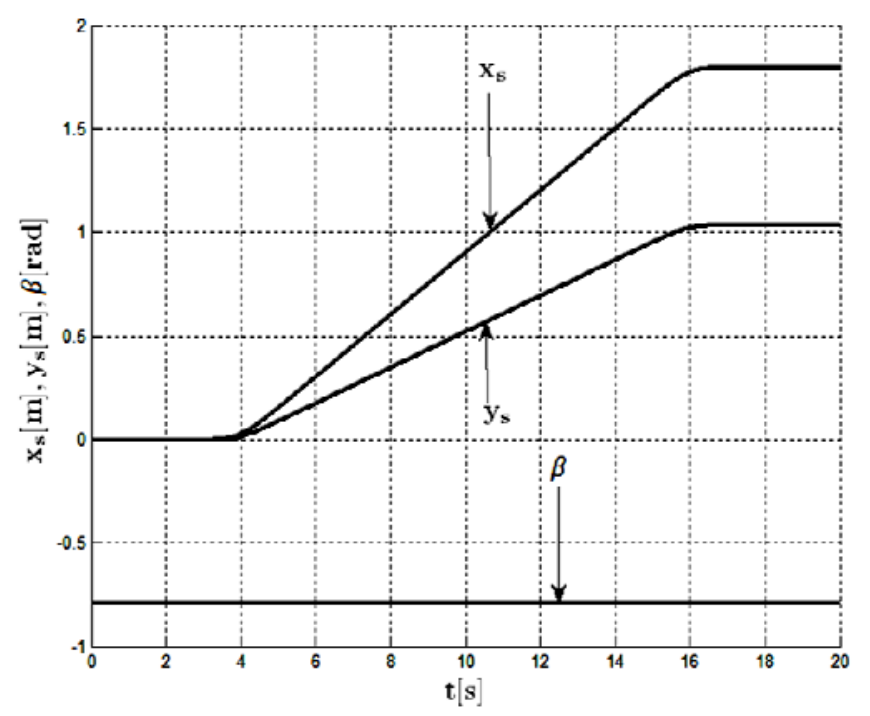

Fig.9. Behaviours of the set kinematic parameters of the characteristic point of the WMR: $x_{s}(t), y_{s}(t)$ and $\beta(t)$. 


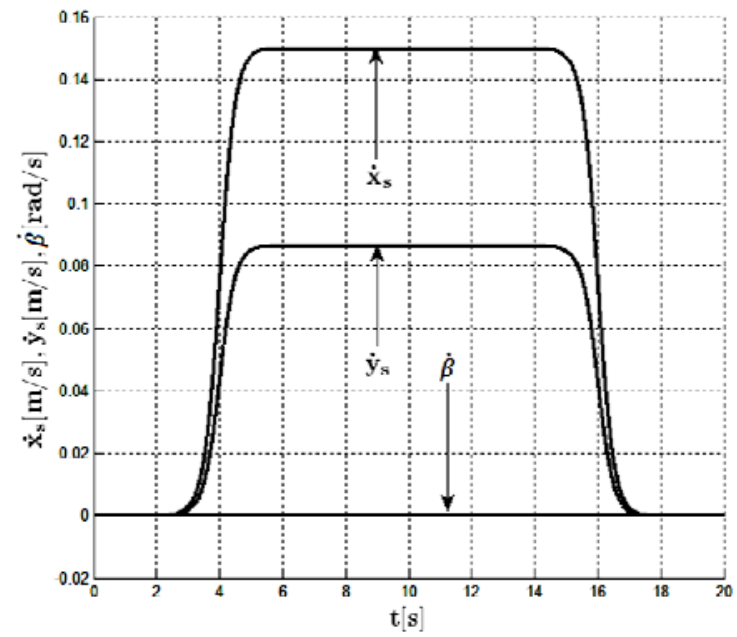

Fig.10. Behaviours of the set kinematic parameters of the characteristic point of the WMR: $\dot{x}_{s}(t), \dot{y}_{s}(t)$ and $\dot{\beta}(t)$.

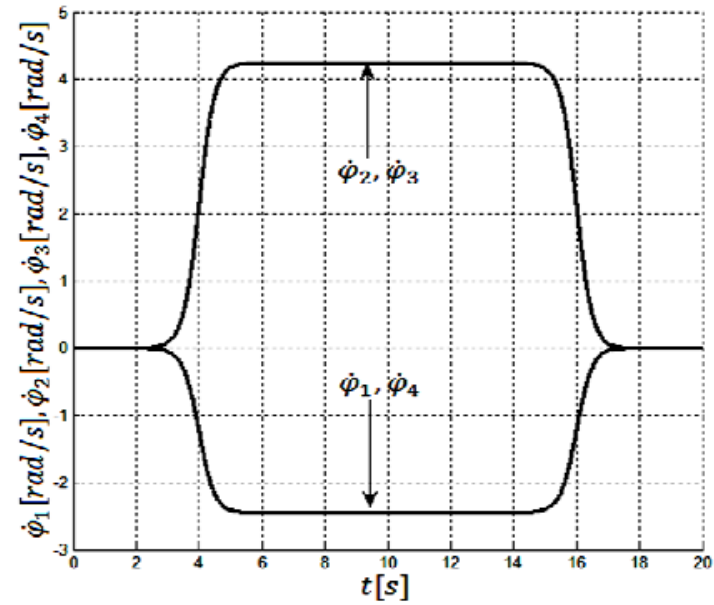

Fig.11. Behaviours of angular velocities of wheels no. 1, 2, 3, 4

Figure 12 presents angular accelerations of the wheels $\ddot{\varphi}_{i}(i=1,2,3,4)$. Figure 13 in turn presents the solution of the inverse dynamics problem, which for the discussed case of motion of the characteristic point of the WMR are two behaviours of driving moments of mecanum wheels identical for the pairs of wheels no. 1,4 and 2,3 .

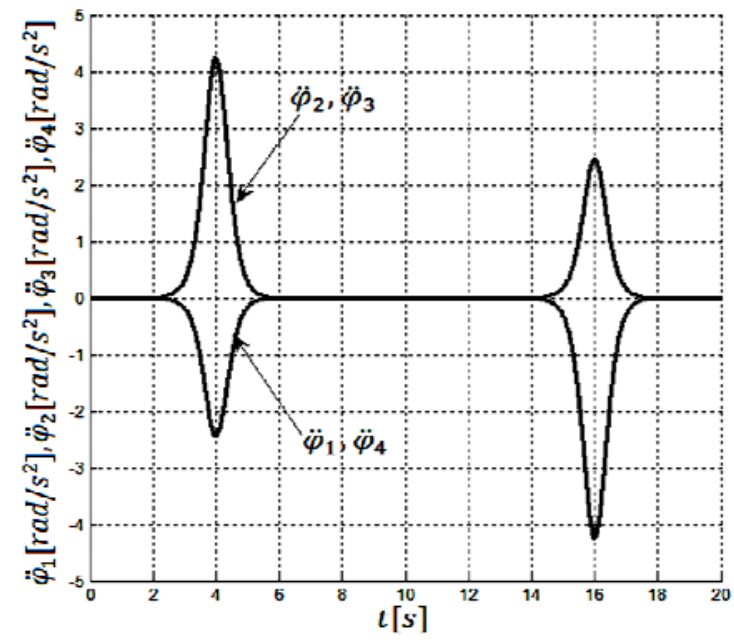

Fig.12. Behaviours of angular accelerations of wheels no. 1, 2, 3, 4. 


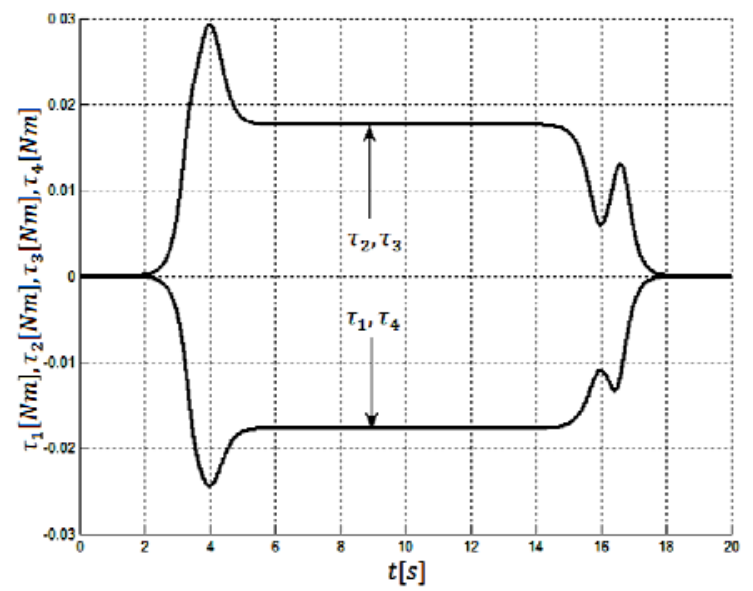

Fig.13. Behaviours of driving moments of wheels no. 1, 2, 3, 4.

For the purpose of verification of the correctness of the derived dynamic equations of motion, a forward dynamics problem has been solved, results of which are illustrated in Fig.14 and Fig.15.

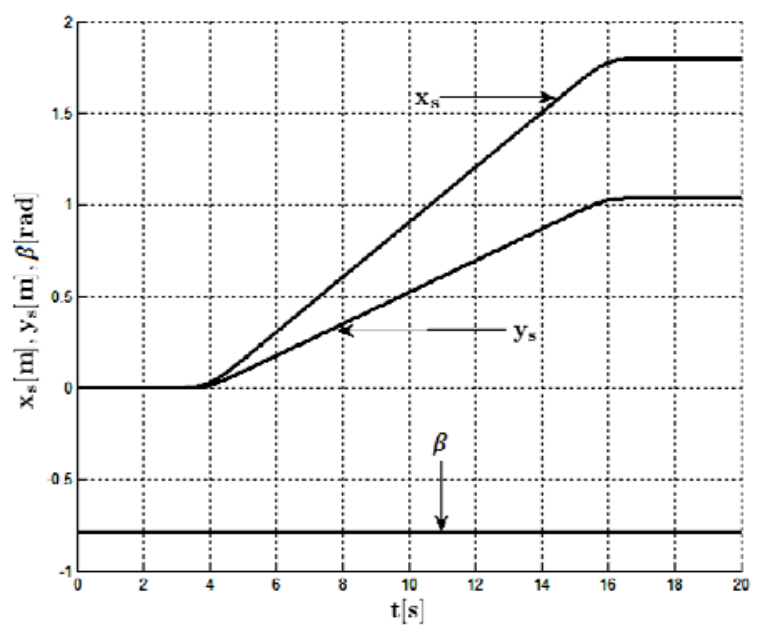

Fig.14. Solution of a forward dynamics problem: behaviours: $x_{s}(t), y_{s}(t)$ and $\beta(t)$.

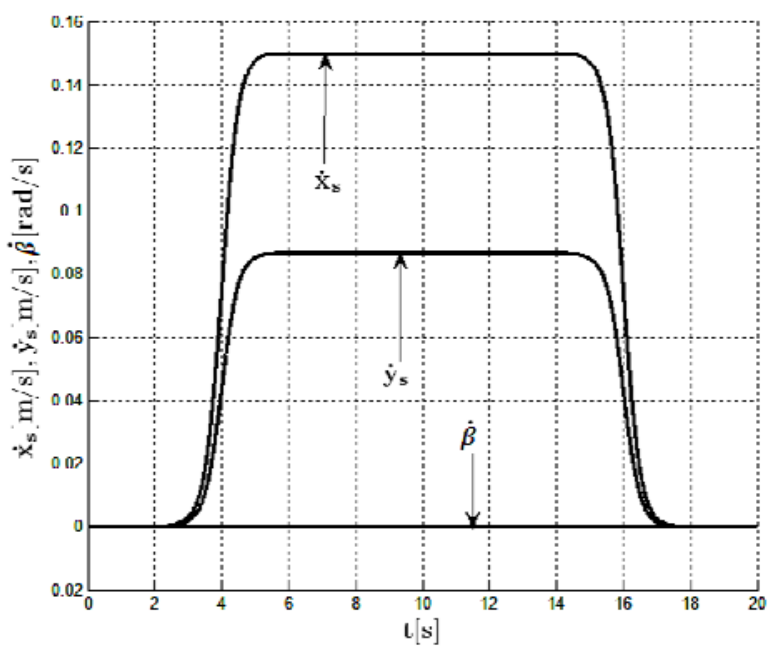

Fig.15. Solution of a forward dynamics problem: behaviours $\dot{x}_{s}(t), \dot{y}_{s}(t)$ and $\dot{\beta}(t)$. 
The difference between the set kinematic behaviours (Figs 9 and 10), and the kinematic behaviours obtained as a result of solution of a forward dynamics problem (Figs 14 and 15) is presented graphically in Figs 16 and 17.

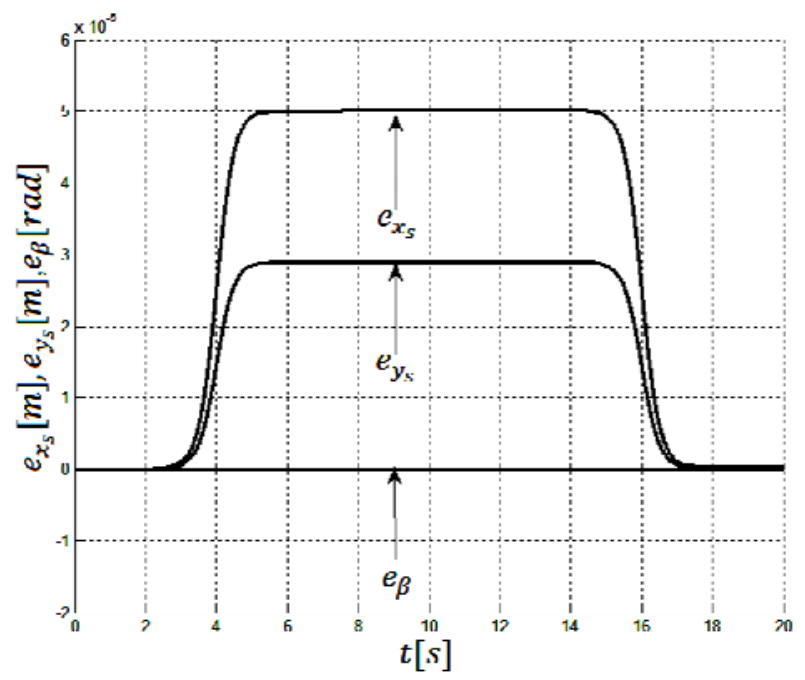

Fig.16. Calculation errors of coordinates of the characteristic point of the WMR: $e_{x_{s}}(t), e_{y_{s}}(t)$ and the angle of rotation of the frame $e_{\beta}(t)$.

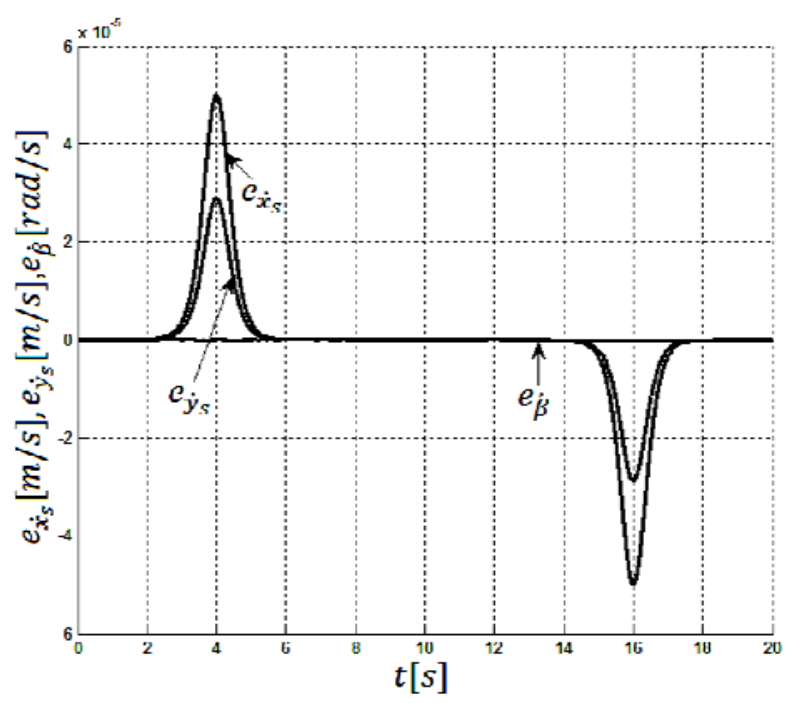

Fig.17. Calculation errors of the projections of velocity of the characteristic point of the WMR: $e_{\dot{x}_{s}}(t), e_{\dot{y}_{s}}(t)$ and the angular speed of the frame $e_{\dot{\beta}}(t)$.

Maple V software for symbolic operations has been used in the course of generation of the mathematical description of the WMR. 


\section{Conclusions}

This work contains an analysis of the dynamics of a four-wheeled WMR with mecanum wheels with the use of Lagrange equations of the second kind without multipliers. The mentioned formalism enables the creation of a description of the dynamics of the object in the form that allows its application in the construction of control systems and in simulation studies. The presented analysis of the dynamics of WMR was conducted for the purpose of a synthesis of tracked motion control algorithms for the discussed object.

The conducted theoretical considerations allowed numerical simulations presenting a solution of the inverse dynamics problem for the motion of the characteristic point of the discussed object on the set trajectory with the determined configuration of the platform. In order to verify the correctness of the obtained solutions, the results of the forward dynamics problem are also presented, which are in turn compared with the set behaviours. As a result, small errors were obtained (order of magnitude $10^{-5}[\mathrm{~m}]$ ), which confirmed the correctness of the entirety of numerical studies and the correctness of the derived dynamic equations of motion for the analysed object.

\section{Nomenclature}

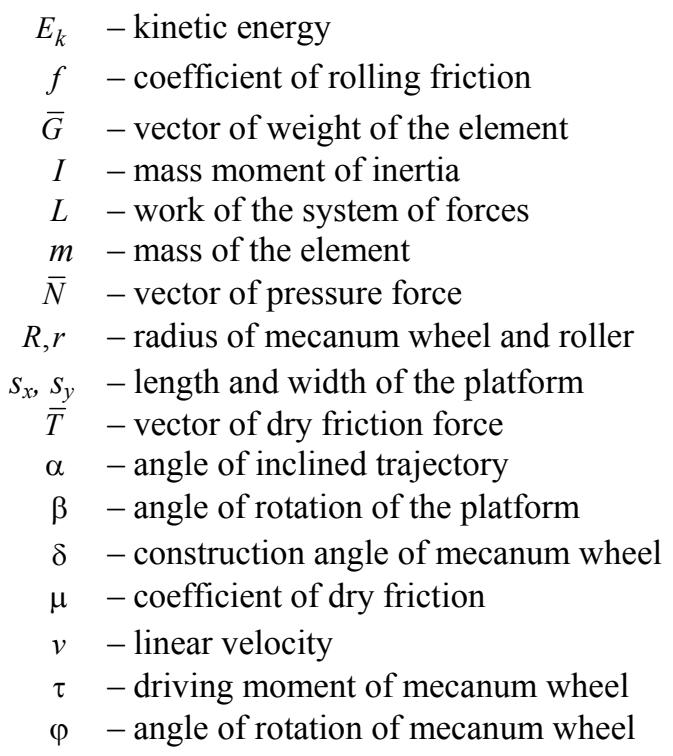

\section{References}

[1] Abdelrahman M., Zeidis I., Bondarev O., Adamov B., Becker F. and Zimmermann K. (2014): A description of the dynamics of a four wheel mecanum mobile system as a basis for a platform concept for special purpose vehicles for disabled persons. - 58-th Ilmenau Scientific Colloquium.

[2] Becker F., Bondarev O., Zeidis I., Zimmermann K., Abdelrahman M. and Adamov B. (2014): An approach to the kinematics and dynamics of a four-wheeled mecanum vehicles. - Scientific Journal of IFToMM, "Problems of Mechanics', special issue, vol.2, No.55, pp.27-37.

[3] Lih-Chang L. and Hao-Yin S. (2013): Modeling and adaptive control of an omni-mecanum-wheeled robot. Intelligent Control and Automation, vol.4, No.2, p.166.

[4] Yunanm Z., Shuangshuang W., Jian Z., Quan S. and Jianmin G. (2010): Research on motion characteristic of omnidirectional robot based on mecanum wheel. - Digital Manufacturing and Automation, vol.2, pp.237-241.

[5] Zimmermann K., Zeidis I. and Abdelrahman M. (2014): Chapter dynamics of mechanical systems with mecanum wheels. - In: Applied Non-Linear Dynamical Systems, pp.269-279, Springer International Publishing. 
[6] Hendzel Z. and Rykała Ł. (2015): Description of kinematics of a wheeled mobile robot with mecanum wheels. Modelling in Engineering, vol.26, No.57, pp.5-12.

[7] Hendzel Z. and Żylski W. (2012): General Mechanics: Dynamics. - Rzeszów University of Technology Publishing House, Rzeszów.

[8] Tzafestas S.G. (2013): Introduction to mobile robot control. - Elsevier.

[9] Żylski W. (1996): Kinematics and dynamics of wheeled mobile robots. - Rzeszów University of Technology Publishing House, Rzeszów.

[10] Burghardt A. (2010): Modelling of dynamic mobile robot using a appell equation. - Acta Mechanica et Automatica, vol.4, No.1, pp.9-12.

[11] Giergiel J.M., Hendzel Z. and Żylski W. (2002): Modeling and control of wheeled mobile robots. - Warsaw: PWN.

[12] Trojnacki M. (2013): Modeling of dynamics of wheeled moile robots. - Warsaw: PIAP.

[13] Wampfler G., Salecker M. and Wittenburg J. (1989): Kinematics, dynamics, and control of omnidirectional vehicles with mecanum wheels. - Mechanics Based Design of Structures and Machines, vol.17, No.2, pp.165-177.

Received: October 16, 2016

Revised: January 19, 2017 